\title{
A Two-dimensional Mapping with a Strange Attractor
}

\author{
M. Hénon \\ Observatoire de Nice, F-06300 Nice, France
}

\begin{abstract}
Lorenz (1963) has investigated a system of three first-order differential equations, whose solutions tend toward a "strange attractor". We show that the same properties can be observed in a simple mapping of the plane defined by: $x_{i+1}=y_{i}+1-a x_{i}^{2}, y_{i+1}=b x_{i}$. Numerical experiments are carried out for $a=1.4, b=0.3$. Depending on the initial point $\left(x_{0}, y_{0}\right)$, the sequence of points obtained by iteration of the mapping either diverges to infinity or tends to a strange attractor, which appears to be the product of a onedimensional manifold by a Cantor set.
\end{abstract}

\section{Introduction}

Lorenz (1963) proposed and studied a remarkable system of three coupled firstorder differential equations, representing a flow in three-dimensional space. The divergence of the flow has a constant negative value, so that any volume shrinks exponentially with time. Moreover, there exists a bounded region $R$ into which every trajectory becomes eventually trapped. Therefore, all trajectories tend to a set of measure zero, called attractor. In some cases the attractor is simply a point (which is then a stable equilibrium point) or a closed curve (known as a limit cycle). But in other cases the attractor has a much more complex structure; it appears to be locally the product of a two-dimensional manifold by a Cantor set. This is known as a strange attractor. Inside the attractor, trajectories wander in an apparently erratic manner. Moreover, they are highly sensitive to initial conditions. These phenomena are of interest for weather prediction (Lorenz, 1963) and more generally for turbulence theory (Ruelle and Takens, 1971; Ruelle, 1975). Further numerical explorations of the Lorenz system have been made by Lanford (1975) and Pomeau (1976).

We present her a "reductionist" approach in which we try to find a model problem which is as simple as possible, yet exhibits the same essential properties as the Lorenz system. Our aim is (i) to make the numerical exploration faster and more accurate, so that solutions can be followed for a longer time, more 


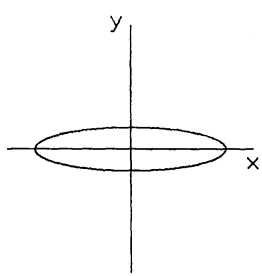

a

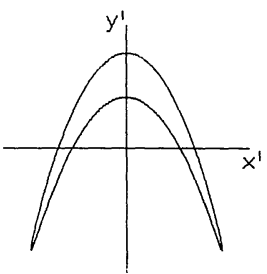

b

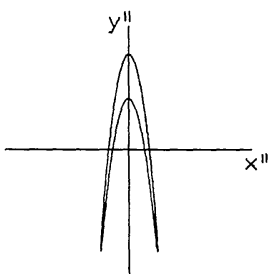

c

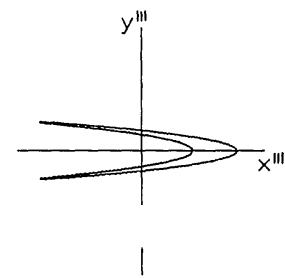

d

Fig. 1. The initial area $a$ is mapped by $T^{\prime}$ into $b$, then by $T^{\prime \prime}$ into $c$, and finally by $T^{\prime \prime}$ into $d$

detailed explorations can be conducted, etc.; (ii) to provide a model which might lend itself more easily to mathematical analysis.

\section{The Model}

Our first step is classical (Birkhoff, 1917) and consists in considering not the whole trajectories in the three-dimensional space, but only their successive intersections with a two-dimensional surface of section $S$. We define a mapping $T$ of $S$ into itself as follows: given a point $A$ of $S$, we follow the trajectory which originates from $A$ until it intersects $S$ again; this new point is $T(A)$. This mapping is sometimes called a Poincaré map. A trajectory is thus replaced by an infinite set of points in $S$, obtained by repeated application of the mapping $T$. The essential properties of the trajectory are reflected into corresponding properties of the set of points. We have thus formally reduced the problem to the study of a twodimensional mapping.

At this point, however, the only advantage really gained is in clarity of presentation of the results; the actual computation of the mapping still requires the numerical integration of the differential equations. Now comes the second and decisive step: we forget about the differential system, and we define a mapping $T$ by explicit equations, giving directly $T(A)$ when $A$ is known. This of course simplifies the computation drastically. The new mapping $T$ does not any more correspond to the Lorenz system; however, by choosing it carefully we may hope to retain the essential properties which we wish to study. Past experience in the measure-preserving case (see Hénon, 1969, and references therein) has shown indeed that the same features are found in dynamical systems defined by differential equations and in mappings defined as such.

The third step consists in specifying $T$. Here we have been inspired by the numerical results of Pomeau (1976) on the Lorenz system, which show clearly how a volume is stretched in one direction, and at the same time folded over itself, in the course of one revolution. This folding effect has been also described by Ruelle (1975, Fig. 5 and 6). We simulate it by the following chain of three mappings of the $(x, y)$ plane onto itself. Consider a region elongated along the $x$ axis (Fig. 1a). We begin the folding by

$$
T^{\prime}: x^{\prime}=x, \quad y^{\prime}=y+1-a x^{2},
$$


which produces Figure $1 \mathrm{~b} ; \mathrm{a}$ is an adjustable parameter. We complete the folding by a contraction along the $x$ axis:

$$
T^{\prime \prime}: x^{\prime \prime}=b x^{\prime}, \quad y^{\prime \prime}=y^{\prime},
$$

which produces Figure $1 \mathrm{c} ; b$ is another parameter, which should be less than 1 in absolute value. Finally we come back to the orientation along the $x$ axis by

$$
T^{\prime \prime}: x^{\prime \prime \prime}=\gamma^{\prime \prime}, \quad y^{\prime \prime \prime}=x^{\prime \prime},
$$

which results in Figure 1 d.

Our mapping will be defined as the product $T=T^{\prime \prime \prime} T^{\prime \prime} T^{\prime}$. We write now $\left(x_{i}, y_{i}\right)$ for $(x, y)$ and $\left(x_{i+1}, y_{i+1}\right)$ for $\left(x^{\prime \prime \prime}, y^{\prime \prime \prime}\right)$ (as a reminder that the mapping will be iterated) and we have

$$
T: x_{i+1}=y_{i}+1-a x_{i}^{2}, \quad y_{i+1}=b x_{i} .
$$

This mapping has some interesting properties. Its Jacobian is a constant:

$$
\frac{\partial\left(x_{i+1}, y_{i+1}\right)}{\partial\left(x_{i}, y_{i}\right)}=-b \text {. }
$$

The geometrical interpretation is quite simple: $T^{\prime}$ preserves areas; $T^{\prime \prime \prime}$ also preserves areas but reverses the sign; and $T^{\prime \prime}$ contracts areas, multiplying them by the constant factor $b$. The property (5) is welcome because it is the natural counterpart of the constant negative divergence in the Lorenz system.

A polynomial mapping satisfying (5) is known as an entire Cremona transformation, and the inverse mapping is also given by polynomials (Engel, 1955, 1958). Indeed we have here

$$
T^{-1}: x_{i}=b^{-1} y_{i+1}, \quad y_{i}=x_{i+1}-1+a b^{-2} y_{i+1}^{2} .
$$

Thus $T$ is a one-to-one mapping of the plane onto itself. This is also a welcome property, because it is the natural counterpart of the fact that in the Lorenz system there is a unique trajectory through any given point.

The selection of $T$ could have been approached in a different way, by looking for the "simplest" non-trivial mapping. It is natural then to consider polynomial mappings of progressively increasing order. Linear mappings are trivial, so the polynomials must be at least of degree 2 . The most general quadratic mapping is

$$
\begin{aligned}
& x_{i+1}=f+a x_{i}+b y_{i}+c x_{i}^{2}+d x_{i} y_{i}+e y_{i}^{2}, \\
& y_{i+1}=f^{\prime}+a^{\prime} x_{i}+b^{\prime} y_{i}+c^{\prime} x_{i}^{2}+d^{\prime} x_{i} y_{i}+e^{\prime} y_{i}^{2}
\end{aligned}
$$

and depends on 12 parameters. But if we impose the condition that the Jacobian is a constant, some relations must be satisfied by these parameters. We can further reduce the number of parameters by an appropriate linear change of coordinates in the plane. In this way, by a slight extension of the results of Engel (1958), it can be shown that the general form (7) is reducible to a "canonical form" depending on two parameters only. This is a generalization of our earlier result (Hénon, 1969) that a quadratic area-preserving mapping can be brought into a form depending on one parameter only. The canonical form can be written in several different ways; and one of them turns out to be identical with (4), which is 
thus reached by an entirely different road! The mapping (4), which was initially constructed in empirical fashion, is in fact the most general quadratic mapping with constant Jacobian.

One difference with the Lorenz problem is that the successive points obtained by repeated application of $T$ do not always converge towards an attractor; sometimes they "escape" to infinity. This is because the quadratic term in (4) dominates when the distance from the origin becomes large. However, for particular values of $a$ and $b$ it is still possible to prove the existence of a bounded "trapping region" $R$, from which the points can never escape once they have entered it (see below Section 5).

$T$ has two invariant points, given by

$$
x=(2 a)^{-1}\left[-(1-b) \pm \sqrt{(1-b)^{2}+4 a}\right], \quad y=b x .
$$

These points are real for

$$
a>a_{0}=(1-b)^{2} / 4 \text {. }
$$

When this is the case, one of the points is always linearly unstable, while the other is unstable for

$$
a>a_{1}=3(1-b)^{2} / 4 \text {. }
$$

\section{Choice of Parameters}

We select now particular values of $a$ and $b$ for a numerical study. $b$ should be small enough for the folding described by Figure 1 to occur really, yet not too small if one wishes to observe the fine structure of the attractor. The value $b=0.3$ was found to be adequate. A good value of $a$ was found only after some experimenting. For $a<a_{0}$ or $a>a_{3}$, where $a_{0}$ is given by (9) and $a_{3}$ is of the order of 1.55 for $b=0.3$, the points always escape to infinity: apparently there exists no attractor in these cases. For $a_{0}<a<a_{3}$, depending on the initial values $\left(x_{0}, y_{0}\right)$, either the points escape to infinity or they converge towards an attractor, which appears to be unique for a given value of $a$. We concentrate now on this attractor. For $a_{0}<a<a_{1}$, where $a_{1}$ is given by (10), the attractor is the stable invariant point. When $a$ is increased over $a_{1}$, at first the attractor is still simple and consists of a periodic set of $p$ points. (An equivalent attractor in the Lorenz problem would be a limit cycle intersecting the surface of section $p$ times). The value of $p$ increases through successive "bifurcations" as $a$ increases, and appears to tend to infinity as a approaches $a$ critical value $a_{2}$, of the order of 1.06 for $b=0.3$. For $a_{2}<a<a_{3}$, the attractor is no more simple, and the behaviour of the points becomes erratic. This is the case in which we are interested. We adopt the following values:

$$
a=1.4, \quad b=0.3 \text {. }
$$

\section{Numerical Results}

Figure 2 shows the result of plotting 10000 successive points, obtained by iteration of $T$, starting from the arbitrarily chosen initial point $x_{0}=0, y_{0}=0$; the vertical scale is enlarged to give a better picture. Figure 3 shows the result of 10000 


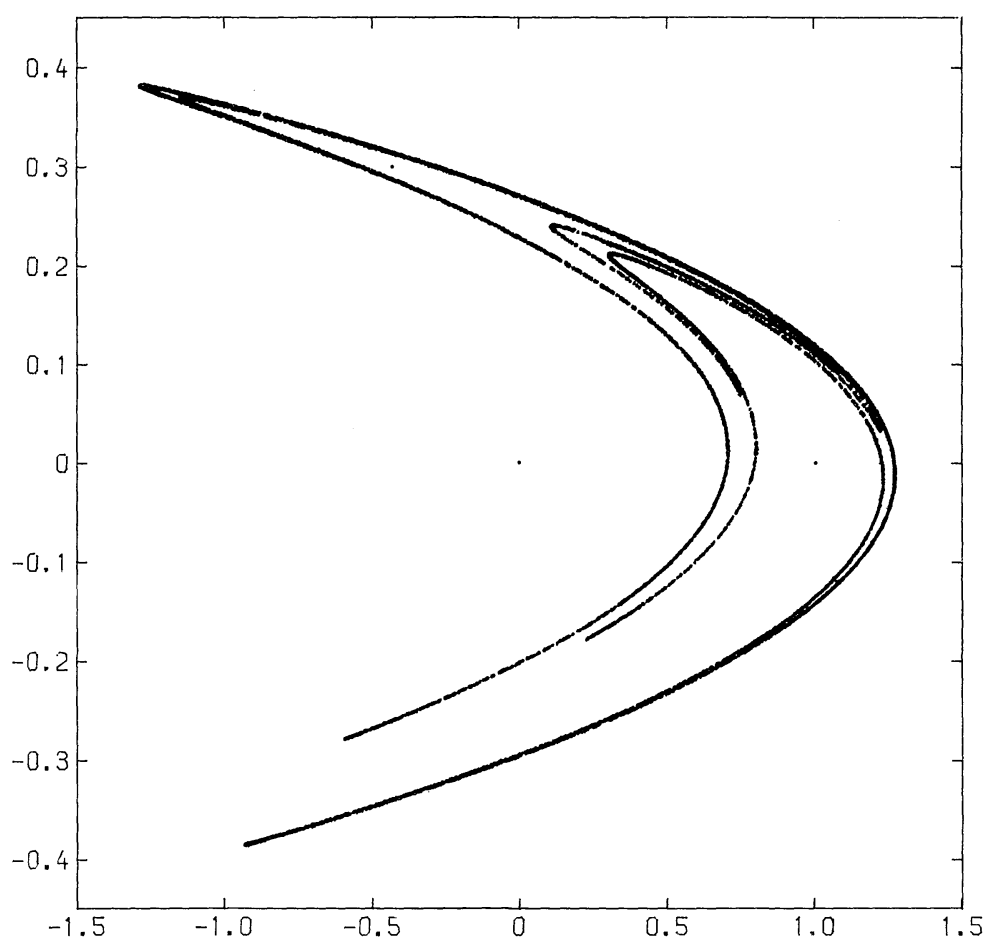

Fig. 2. 10000 successive points obtained by iteration of the mapping $T$ starting from $x_{0}=0, y_{0}=0$

iterations of $T$ again, starting from a different point: $x_{0}=0.63135448, y_{0}=$ 0.18940634 (this choice will be explained below). The two figures are seen to be almost identical. This suggests strongly that what we see in both figures is essentially the attractor itself: the successive points quickly approach the attractor and soon become undistinguishable from it at the scale of the figure. This is confirmed if one looks at the first few points on Figure 2. The initial point at $x_{0}=0, y_{0}=0$ and the first iterate at $x_{1}=1, y_{1}=0$ are clearly visible; the second iterate is still visible at $x_{2}=-0.4, y_{2}=0.3$; the third iterate can barely be distinguished at $x_{3}=1.076, y_{3}=-0.12$; and the fourth iterate at $x_{4}=-0.7408864$, $y_{4}=0.3228$ is already lost inside the attractor at the resolution of Figure 2 . The following points then wander over the attractor in an apparently erratic manner.

One of the two unstable invariant points has the coordinates, given by (8):

$$
x=0.63135448 \ldots, \quad y=0.18940634 \ldots .
$$

This point appears to belong to the attractor. The two eigenvalues $\lambda_{1}, \lambda_{2}$ and the slopes $p_{1}, p_{2}$ of the corresponding eigenvectors are

$$
\begin{aligned}
& \lambda_{1}=0.15594632 \ldots, \quad p_{1}=1.92373886 \ldots, \\
& \lambda_{2}=-1.92373886 \ldots, \quad p_{2}=-0.15594632 \ldots .
\end{aligned}
$$

The instability is due to $\lambda_{2}$. The corresponding slope $p_{2}$ appears to be tangent to the "curves" in Figure 2. 


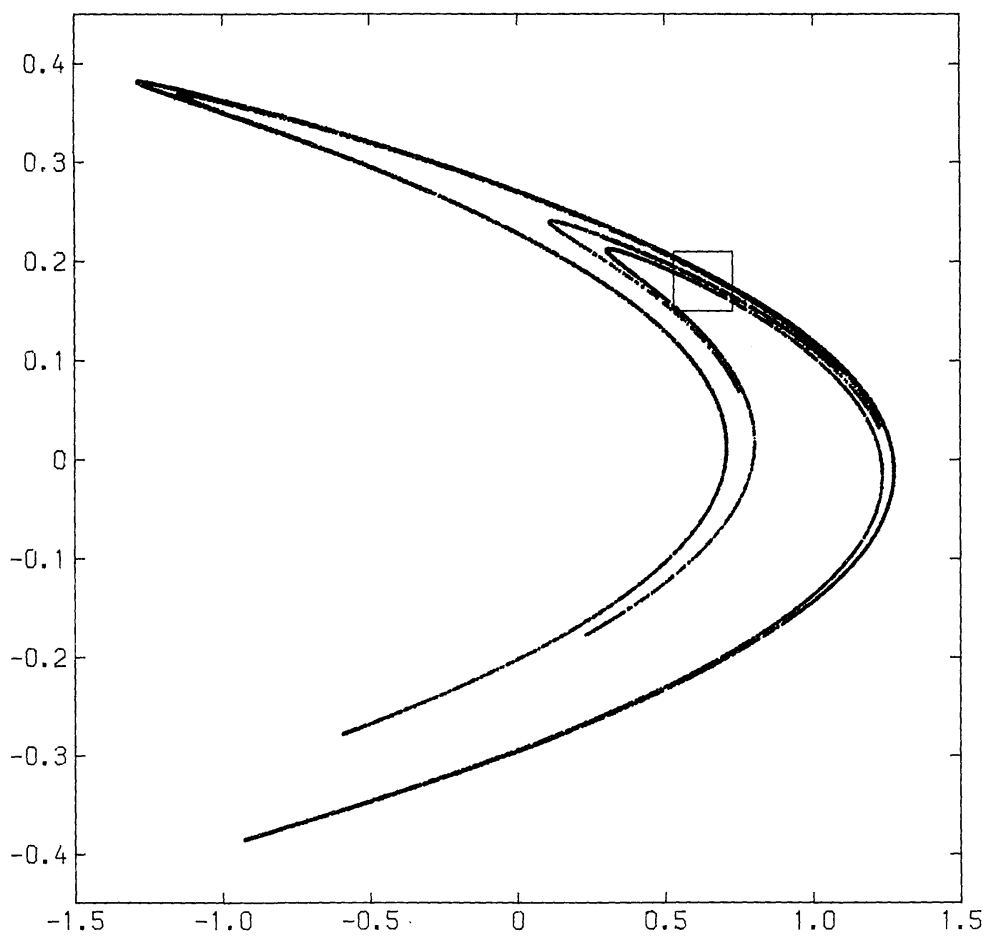

Fig. 3. Same as Figure 2, but starting from $x_{0}=0.63135448, y_{0}=0.18940634$

These properties allow us to eliminate the "transient regime" in which the points approach the attractor, and which is not of much interest: we simply start from the close vicinity of the unstable point (12), by rounding off its coordinates to 8 digits. This is done in Figure 3 and in the following figures. The points quickly move away along the line of slope $p_{2}$ since $\left|\lambda_{2}\right|$ is appreciably larger than 1 .

The attractor appears to consist of a number of more or less parallel "curves"; the points tend to distribute themselves densely over these curves. The few gaps that can still be seen on Figures 2 and 3 have probably no particular significance. Their locations are not the same on the two figures. They are simply due to statistical fluctuations in the quasi-random distribution of points, and they would disappear if more moints were plotted. Thus, the longitudinal structure of the attractor (along the curves) appears to be simple, each curve being essentially a one-dimensional manifold.

The transversal structure (across the curves) appears to be entirely different, and much more complex. Already on Figures 2 and 3 a number of curves can be seen, and the visible thickness of some of them suggests that they have in fact an underlying structure. Figure 4 is a magnified view of the small square of Figure 3: some of the previous "curves" are indeed resolved now into two or more components. The number $n$ of iterations has been increased to $10^{5}$, in order to have a sufficient number of points in the small region examined. The small square in Figure 4 is again magnified to produce Figure 5, with $n$ increased to $10^{6}$ : again the 


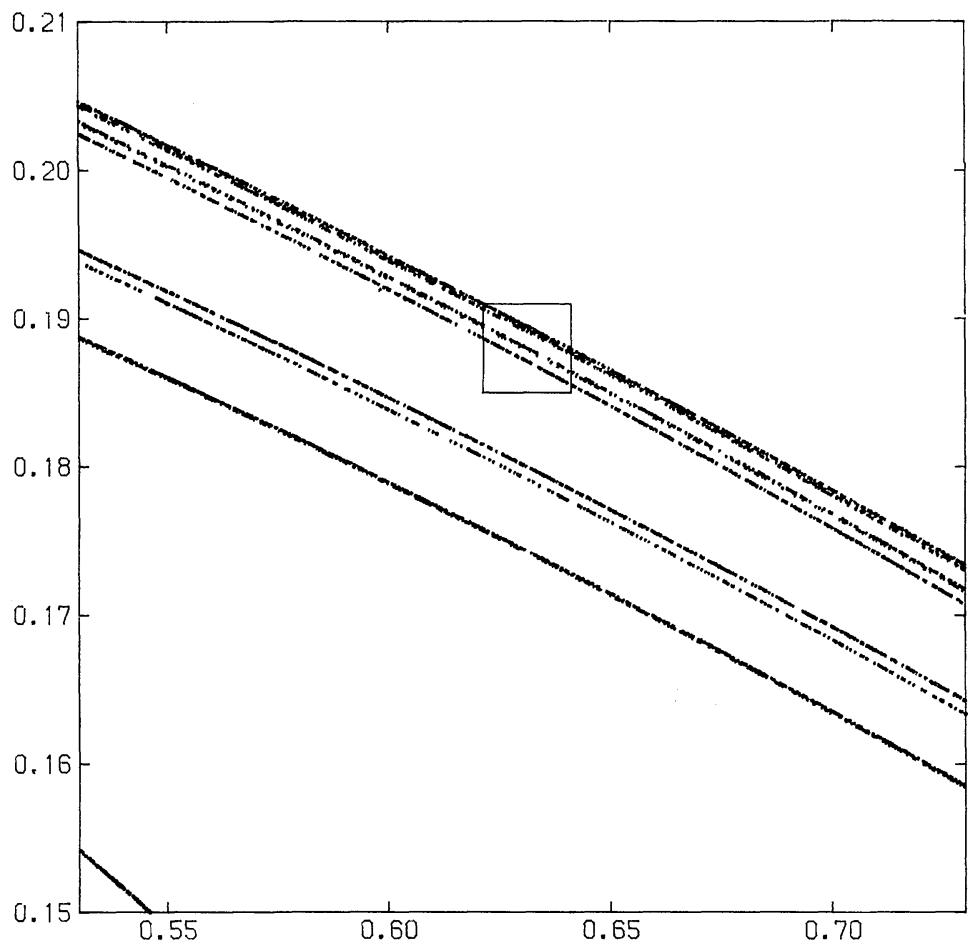

Fig. 4. Enlargement of the squared region of Figure 3. The number of computed points is increased to $n=10^{5}$

number of visible "curves" increases. One more enlargement results in Fig. 6, with $n=5 \times 10^{6}$ : the points become sparse but new curves can still easily be traced.

These figures strongly suggest that the process of multiplication of "curves" will continue indefinitely, and that each apparent "curve" is in fact made of an infinity of quasi-parallel curves. Moreover, Figures 4 to 6 indicate the existence of a hierarchical sequence of "levels", the structure being practically identical at each level save for a scale factor. This is exactly the structure of a Cantor set.

The frames of Figures 4 to 6 have been chosen so as to contain the invariant point (12). This point appears to lie on the upper boundary of the attractor. Surprisingly, its presence is completely invisible on the figures; this contrasts with the area-preserving case, were stable and unstable invariant points play a very conspicuous role (see for instance Hénon, 1969). On the other hand, the presence of the invariant point explains, locally at least, the hierarchy of similar structures: at each application of the mapping, the scale of the transversal structure is multiplied by $\lambda_{1}$ given by (13). At the same time, the points spread out along the curves, as dictated by the value of $\lambda_{2}$.

\section{A Trapping Region}

The fact that even after $5 \times 10^{6}$ iterations the points have not diverged to infinity suggests that there is a region of the plane from which the points cannot escape. 


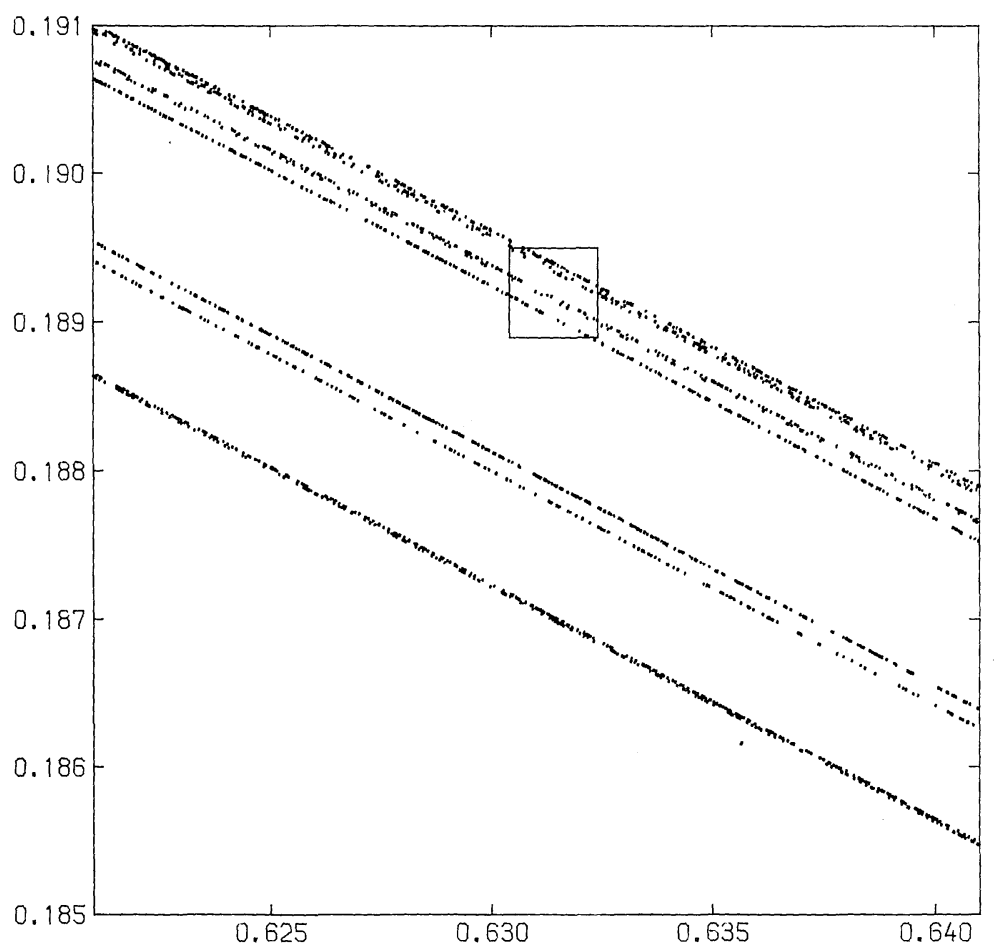

Fig. 5. Enlargement of the squared region of Figure $4 ; n=10^{6}$

This can be actually proved by finding a region $R$ which is mapped inside itself. An example of such a region is the quadrilateral $A B C D$ defined by

$$
\begin{array}{llll}
x_{A}=-1.33, & y_{A}=0.42, & x_{B}=1.32, & y_{B}=0.133, \\
x_{C}=1.245, & y_{C}=-0.14, & x_{D}=-1.06, & y_{D}=-0.5 .
\end{array}
$$

The image of $A B C D$ is a region bounded by four arcs of parabola, and it can be shown by elementary algebra that this image lies inside $A B C D$. Plotting the quadrilateral on Figure 2 or 3 , one can verify that it encloses the observed attractor.

\section{Conclusions}

The simple mapping (4) appears to have the same basic properties as the Lorenz system. Its numerical exploration is much simpler: in fact most of the exploratory work for the present paper was carried out with a programmable pocket computer (HP-65). For the more extensive computations of Figures 2 to 6, we used a IBM 7040 computer, with 16-digit accuracy. The solutions can be followed over a much longer time than in the case of a system of differential equations. The accuracy is also increased since there are no integration errors.

Lorenz (1963) inferred the Cantor-set structure of the attractor from reasoning, but could not observe it directly because the contracting ratio after one "circuit" 


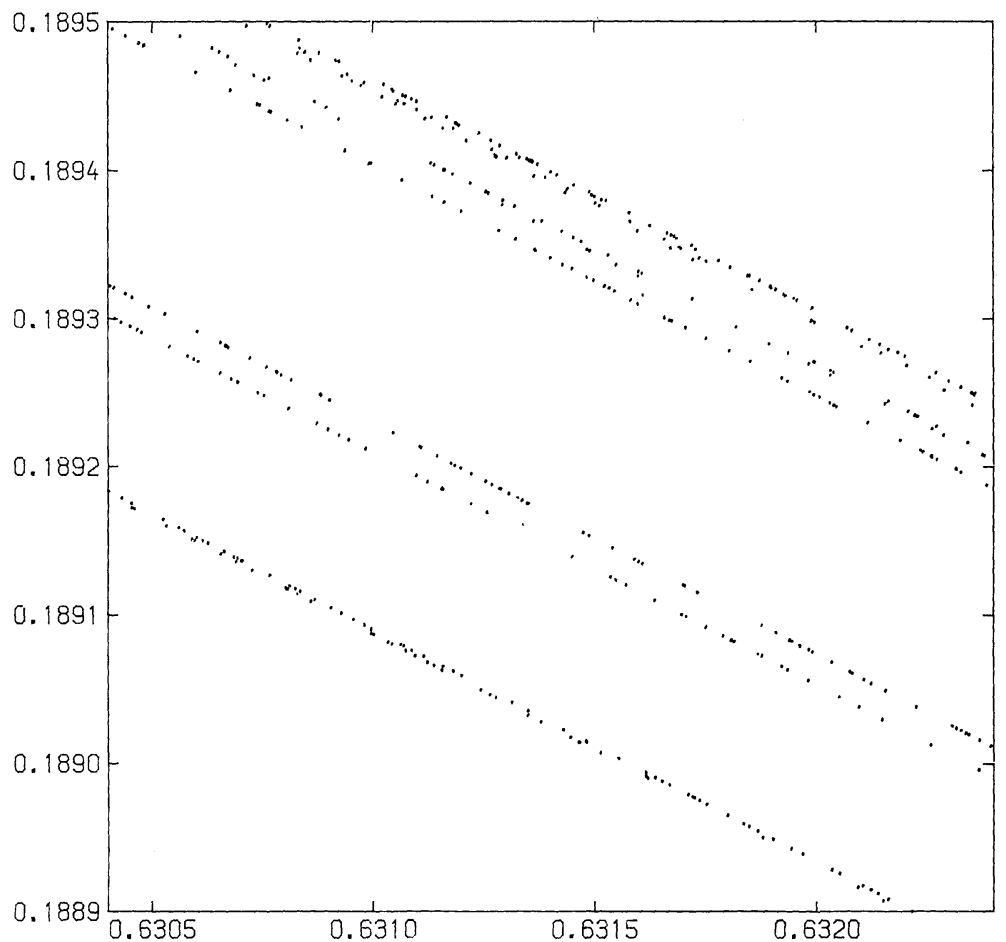

Fig. 6. Enlargement of the squared region of Figure $5 ; n=5 \times 10^{6}$

was too small: $7 \times 10^{-5}$. A similar experience was reported by Pomeau (1976). In the present mapping, the contracting ratio after one iteration is 0.3 , and one can easily observe a number of successive levels in the hierarchy. This is also facilitated by the larger number of points.

Finally, for mathematical studies the mapping (4) might also be easier to handle than a system of differential equations.

\section{References}

Birkhoff, G. D. : Trans. Amer. Math. Soc. 18, 199 (1917)

Engel, W.: Math. Annalen 130, 11 (1955)

Engel, W.: Math. Annalen 136, 319 (1958)

Hénon, M.: Quart. Appl. Math. 27, 291 (1969)

Lanford, O.: Work cited by Ruelle, 1975

Lorenz, E. N.: J. atmos. Sci. 20, 130 (1963)

Pomeau, Y.: to appear (1976)

Ruelle, D., Takens, F.: Comm. math. Phys. 20, 167; 23, 343 (1971)

Ruelle, D.: Report at the Conference on "Quantum Dynamics Models and Mathematics" in Bielefeld, September 1975

Communicated by K. Hepp 
